\title{
Delaying orthostatic syncope with mental challenge: A pilot study
}

\author{
Nandu Goswami a,*, Andreas Roessler a , Helmut Hinghofer-Szalkay a,b, \\ Jean-Pierre Montani ${ }^{c}$, Andrew Steptoe ${ }^{\mathrm{d}}$ \\ a Institute of Physiology, Medical University of Graz, Austria \\ b Institute of Adaptive and Spaceflight Physiology, Graz, Austria \\ c Department of Medicine/Physiology, University of Fribourg, Switzerland \\ ${ }^{\mathrm{d}}$ University College, London, UK
}

\begin{abstract}
At orthostatic vasovagal syncope there appears to be a sudden decline of sympathetic activity. As mental challenge activates the sympathetic system, we hypothesized that doing mental arithmetic in volunteers driven to the end point of their cardiovascular stability may delay the onset of orthostatic syncope. We investigated this in healthy male subjects. Each subject underwent a head up tilt (HUT) + graded lower body negative pressure (LBNP) up to presyncope session (control) to determine the orthostatic tolerance time, OTT (Time from HUT commencement to development of presyncopal symptoms/signs). Once the tolerance time was known, a randomized crossover protocol was used: either 1) Repeat HUT + LBNP to ensure reproducibility of repeated run or 2) HUT + LBNP run but with added mental challenge ( 2 min before the expected presyncope time). Test protocols were separated by 2 weeks. Our studies on five male test subjects indicate that mental challenge improves orthostatic tolerance significantly. Additional mental loading could be a useful countermeasure to alleviate the orthostatic responses of persons, particularly in those with histories of dizziness on standing up, or to alleviate hypotension that frequently occurs during hemodialysis or on return to earth from the spaceflight environment of microgravity.
\end{abstract}

\section{Introduction}

Orthostatic stress constitutes a serious problem in persons with histories of dizziness upon standing up. In such persons, the cardiovascular system may not always be able to maintain mean arterial pressure, thus leading to a critical decrease in cerebral blood flow and consequently syncope. Syncope is the end-point of orthostatic stability and is defined as transient, self-limiting loss of consciousness. Despite intensive research, orthostatic intolerance remains a problem.

At orthostatic vasovagal syncope there appears to be a sudden withdrawal of sympathetic activity [1]. Any activity that increases the sympathetic activity in subjects reaching orthostatic syncope, at least hypothetically speaking, should lead to improvements in orthostatic capacity. Therefore, maneuvers such as handgrip, cold-water immersion or mental challenge, which result in increases in sympathetic activity, should affect the orthostatic lability. As handgrip and cold-water immersion may not be available on the bedside or roadside, we chose mental challenge application for our study.

\footnotetext{
* Corresponding author at: Institute of Physiology Harrachgasse 21/5, Medical University of Graz, 8010 Graz, Austria. Tel.: + 433163804278 .

E-mail address: Nandu.goswami@medunigraz.at (N. Goswami).
}

\subsection{Mental challenge and interaction with orthostatic stress}

The neurovascular response to mental challenge [2] includes activation of the sympathetic system, vasoconstriction in the splanchnic and renal regions but vasodilatation in skeletal muscles [3], resulting in increases in heart rate, cardiac output and blood pressure.

In daily life, we subject our body to both mental and physical stresses. The interaction between these stressors when applied together, however, is poorly understood. Since the mechanisms of cardiovascular regulation have been reported to be different in orthostatic and mental stresses, we previously investigated the modulation of head up tiltinduced orthostatic responses by mental challenge, applied using mental arithmetic [4]. We observed that mental arithmetic (adding or subtracting six or seven from two or three digit numbers) increased mean arterial pressure and prevented the decrease in cardiac output when it was done while standing up [4].

We hypothesized that doing mental challenge while standing may delay the onset of orthostatic syncope. We investigated this in healthy male subjects; in each of these presyncope was induced. Mental challenge was applied using mental arithmetic: Participants were asked to add or subtract continuously the numbers 6 or 7, randomly, from a 2 or 3 digit numbers and to state the correct answers. In study [4], participants did only 10 min of head up tilt, with and without 10 min mental arithmetic. In the present study, subjects were driven up to presyncope and mental arithmetic was applied only for 2-3 min preceding presyncope. 
The mental arithmetic task has a lot of practical implications as calculations can be performed in all situations and circumstances, even in emergency situations, and without the need for any material or instruction.

\section{Methodology}

\subsection{Participants}

We focused on healthy men whose age and physical characteristics were homogeneous because gender and age may affect orthostatic stress responses [5]. The study was carried out in healthy, non-obese, non-smoking, non-medicated men who were free from any somatic or mental condition. The subjects were free from histories of syncope, cardiovascular disorders, vestibular and somatosensory functions alterations and psychiatric disorders. Our subjects also did not complain of any vestibular symptoms during- or between the three protocols. Participants were familiarized with the test protocol and gave written informed consent to participate in the study. The study was approved by the Graz University Ethics Board and the study performed in accordance with the 1989 WMA Declaration of Helsinki.

\subsection{Study design and administration}

We asked participants to abstain from vigorous exercise, coffee or any stimulants for 2 days before the test sessions. The test persons were fasting and voided before commencement of the experiments.

Each experiment started with a $30 \mathrm{~min}$ supine rest period to acquire cardiovascular steady state conditions. At minute zero, the electronically controlled tilt table was brought to $70^{\circ}$ head-up (HUT) position. $70^{\circ}$ was used as it represents 0.94 sine of tilt angle and about $94 \%$ of orthostatic load on a person [6]. Indeed most laboratories use 60 to $80^{\circ}$ tilt angles to provoke hypotension and bradycardia. After four more minutes, additional $15 \mathrm{~mm} \mathrm{Hg}$ of lower body negative pressure (LBNP) was provided. LBNP was increased by $15 \mathrm{~mm} \mathrm{Hg}$ every $4 \mathrm{~min}$, particularly as we wanted to drive the subjects to presyncope rather quickly. We chose this protocol, as LBNP stress duration influences stress responses [5], and we also wanted to minimize possible effects of rapid adaptation. As soon as presyncopal signs or symptoms occurred, the table was brought back to $0^{\circ}$ and LBNP was stopped at once. The criteria of presyncope were [7]: a) blood pressure drops below systolic $80 \mathrm{~mm} \mathrm{Hg}$ or by $\geq 20 \mathrm{~mm} \mathrm{Hg} / \mathrm{min}$, diastolic by $\geq 10 \mathrm{~mm} \mathrm{Hg} / \mathrm{min}$, and/or heart rate decreases by $\geq 15 \mathrm{bpm}$, b) lightheadedness, dizziness, visual disturbances, nausea, stomach awareness, clammy skin, excessive sweating, or skin pallor.

During the entire protocol subjects were instructed to avoid undue movements of the lower limbs and to breathe normally. Test persons were secured and had access to an emergency shutdown (automatic return to supine and pressure neutralization) at all times.

The test was carried out between 9:00 AM to 1:00 PM in a semidark quiet room, maintained at $24{ }^{\circ} \mathrm{C}$ and humidity at $55 \%$.

\subsection{Protocols}

Each subject underwent a control HUT + LBNP run (PS1) to determine the orthostatic tolerance time, OTT (time from the commencement of HUT to development of presyncopal symptoms or signs). Once the OTT was known, a randomized crossover protocol was used: either 1 ) repeat HUT + LBNP to ensure reproducibility of repeated run (PS2) or 2) HUT + LBNP run but with added mental challenge ( 2 min before the expected presyncope time). Two weeks separated each protocol.

Mental challenge was applied by using mental arithmetic: Participants were asked to add or subtract continuously the numbers 6 or 7, randomly, from a 2 or 3 digit number [4] and to state the correct answer. A new number combination was provided every $5 \mathrm{~s}$ on a computer screen fixed at level of the eye of the participant.

ANOVA was used to compare effects of repeat runs and mental challenge. Results are presented as means \pm standard deviation.

\section{Results}

The study criteria were met by 5 male participants of age $27.2 \pm$ 1.8 years, weight $74 \pm 6.1 \mathrm{~kg}$, height $181.6 \pm 4.9 \mathrm{~cm}$ (mean \pm SD).

Using the HUT + graded LBNP, presyncope was achieved in all the subjects. Table 1 summarizes the averages of orthostatic tolerance (in min) across the three runs.

For PS1, PS2 and PS + MA, average orthostatic tolerance times were $9.07 \pm 4.45,10.03 \pm 5.03$ and $13.29 \pm 3.29$ min, respectively. The addition of mental arithmetic resulted in average improvements in the OTT of $3.73 \pm 2.39 \mathrm{~min}$. By applying mental arithmetic 2 to 3 min before presyncope, we observed increases in orthostatic tolerance. Fig. 1 shows a representative tracing of a male subject, without (Fig. 1a) and with (Fig. 1b) mental arithmetic application.

As seen in Fig. 1a, OTT was 12 min $30 \mathrm{~s}$ in this subject. Addition of mental arithmetic, 2 min before OTT, resulted in increased orthostatic tolerance (18 $\mathrm{min}$ ).

Generally speaking, in the five male test subjects, mental challenge improved orthostatic capacity (Table 1, Fig. 2). For example, PS1 vs. PS + MA as well as PS2 vs. PS + MA showed significant differences ( $\mathrm{p} 0.028,0.034$, respectively) whereas no significant differences were seen between PS1 and PS2 ( $p$ 0.276).

\section{Discussion}

Our pilot results on five male test subjects indicate that mental challenge improves orthostatic tolerance significantly (Fig. 2). Additionally, it appears that mental arithmetic application resulted in greater improvements in orthostatic capacity in subjects with poor orthostatic tolerance.

HUT + LBNP constitutes graded orthostatic stress paradigm, where HUT is followed by graded increases in LBNP until subjects reach presyncope. The advantage of combined graded orthostatic stress is the absence of medication to induce syncope. For example, in nitro-glycerine induced vasovagal syncope studies [8] the effects of this drug on cardiac output make it difficult to ascertain what would be the hemodynamic responses in healthy or non-medicated subjects. Furthermore, intravenous cannulation is avoided, which has been shown to spuriously affect cardiovascular responses and orthostatic tolerance [9]. For details on the combined HUT and graded LBNP protocol, please refer to [10].

\subsection{Interaction of mental and orthostatic stresses: Physiological effects}

We have previously shown that initial effects of mental challenge during orthostatic challenge include greater increases in heart rate

Table 1

Summary of the results of 5 subjects. Column 1: Average of orthostatic tolerance times (OTT, in min) of presyncopal run 1 (PS1), presyncopal run 2 (PS2); Column 2: Improvements in OTT (measured by subtracting from mental arithmetic induced orthostatic tolerance the averages of PS1 and PS2); Column 3: Proportional improvements in OTT derived by dividing improvements in OTT (Column 2) with the corresponding baseline (Column 1).

\begin{tabular}{lll}
\hline $\begin{array}{l}\text { Average OTT } \\
\text { (PS1 and PS2 runs) }\end{array}$ & $\begin{array}{l}\text { Improvement in OTT } \\
\text { [Mental arithmetic OTT } \\
\text { minus Average PS1 and PS2] }\end{array}$ & $\begin{array}{l}\text { Proportional improvements } \\
\text { [Net improvement/baseline] }\end{array}$ \\
\hline 2.117 & 7.133 & 3.370 \\
9.125 & 4.292 & 0.470 \\
9.475 & 2.375 & 0.251 \\
14.075 & 4.158 & 0.295 \\
12.950 & 0.733 & 0.057 \\
\hline
\end{tabular}


a

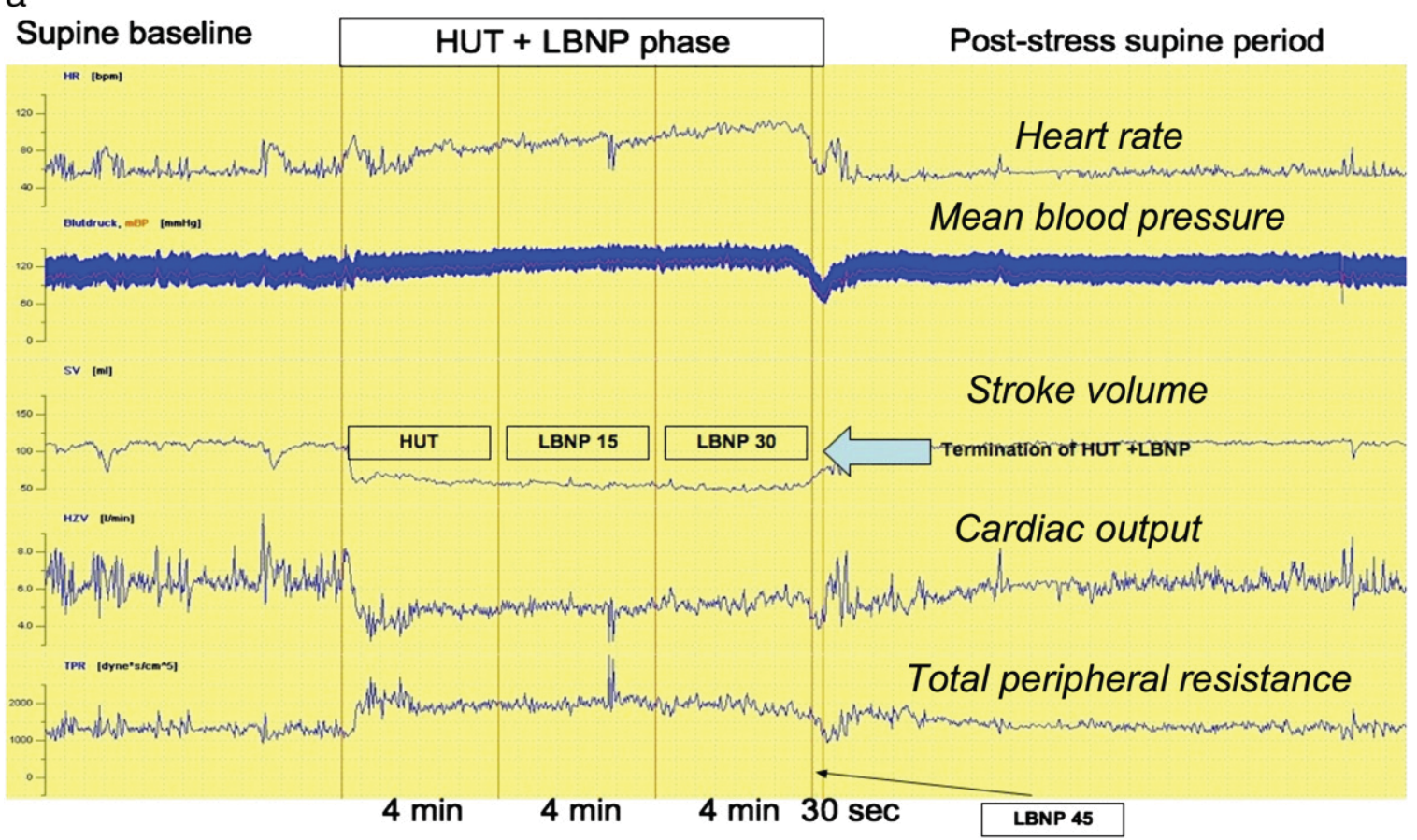

b

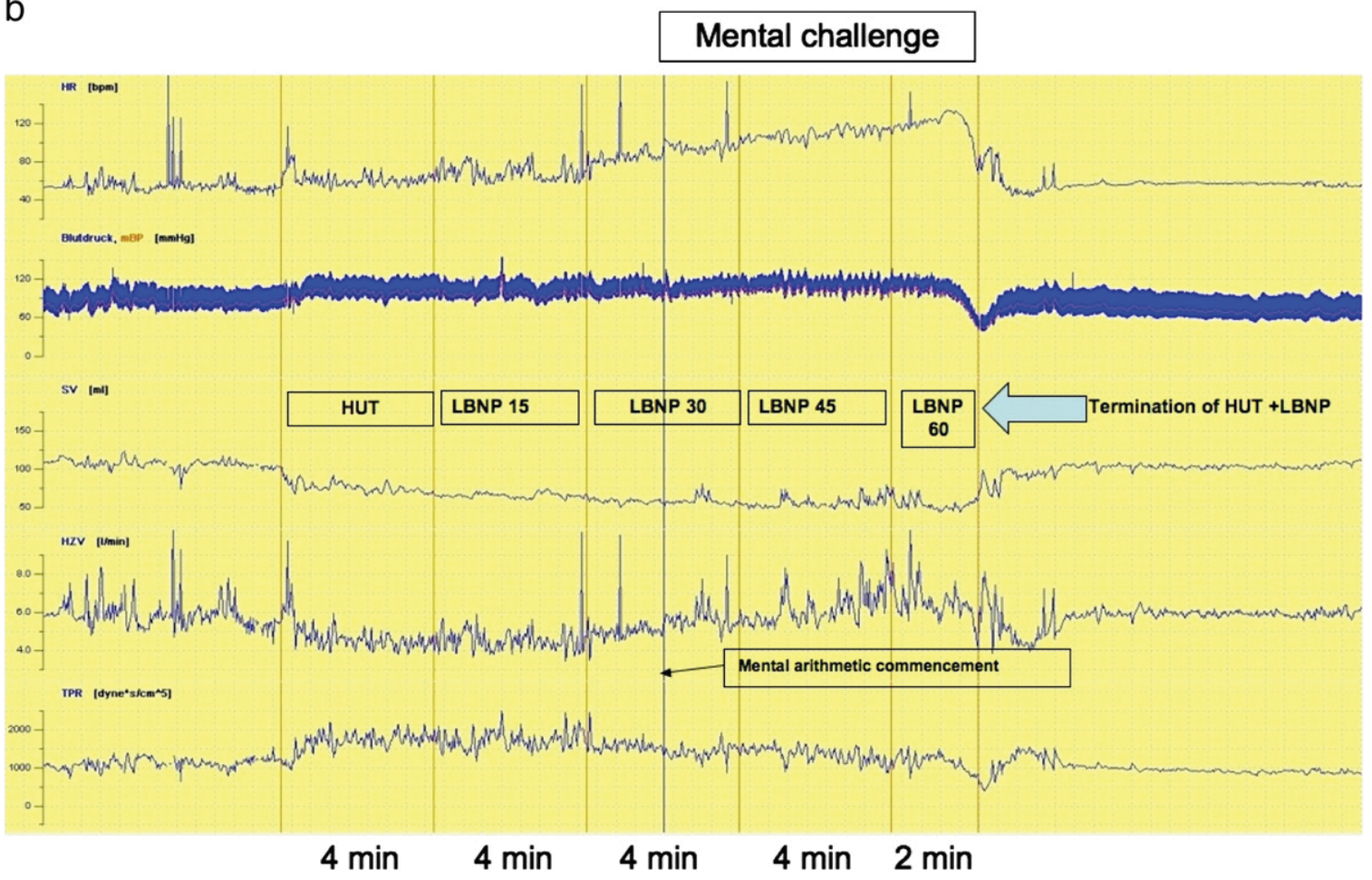

Fig. 1. a. Representative tracing of head up tilt (HUT) and graded lower body negative pressure (LBNP) induced hemodynamic changes in a subject. b. Same subject with added mental arithmetic, which was applied 2 min before the expected orthostatic tolerance time (OTT). Legend: From top to bottom, the measurements represent heart rate (bpm), mean blood pressure $(\mathrm{mm} \mathrm{Hg})$, stroke volume $(\mathrm{ml})$, cardiac output (= heart rate $\times$ stroke volume) and total peripheral resistance $\left(\right.$ dyne.m $^{-2}$ per $\mathrm{cm}^{5}$ ).

than with mental arithmetic (MA) or head up tilt (HUT) alone [4]. As shown in the Fig. 1b), which is a representative tracing of the results obtained in all the five volunteers, mental challenge led to greater increases in heart rate, despite similar reductions in stroke volume such as those during orthostatic stress alone, which was able to maintain the cardiac output. Overall, the cardiac output (representative of global tissue perfusion in healthy populations) decreases were less with combinations of mental and orthostatic challenges in comparison to orthostasis alone.

When comparing effects of the additional mental arithmetic application, applied two minutes before known presyncopal time, no differences in the mean blood pressure were observed. This again confirms that arterial blood pressure is the primary regulated variable during stress [11]. 


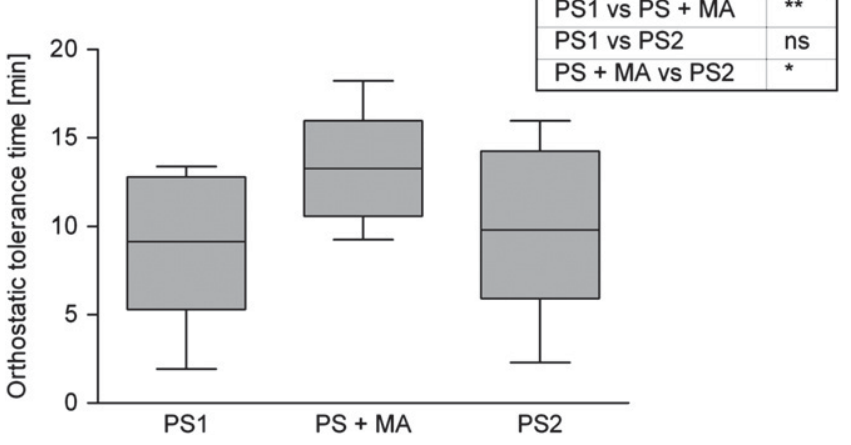

Fig. 2. Box plots of the five males who underwent presyncopal runs, with and withou mental challenge. PS1: Presyncopal session 1, control; PS2: Presyncopal session 2, to assess reproducibility; PS + MA: Presyncopal session with added mental arithmetic. ANOVA was used to compare effects of repeat runs and mental challenge.

Psychological and physical stressors elicit varying physiological responses and both are based on fundamentally different centralautonomic regulatory mechanisms [12]. For example, central hypovolemia induces cardiopulmonary baroreceptor unloading but mental challenge increases central command and arterial baroreceptor loading [13]. The added effects of mental arithmetic (e.g. heart rate increases) in our volunteers, driven to the end point of their cardiovascular stability, may be due to the modulation of baroreflex function by behavior/ mental loading $[14,15]$.

Contradictory responses of total peripheral resistance to mental stress have been reported [16]. We observed significant decreases in total peripheral resistance in response to the mental arithmetic, in agreement with the known vasodilatory effect of mental stress [17]. The latter is mediated by both reduction in sympathetic neural vasoconstriction and beta-adrenergic vasodilatation [17]. It appears that despite reducing the peripheral resistance, mental arithmetic induced heart rate increases, leading to increases in cardiac output, which were sufficient to provide improved orthostatic tolerance.

On the other hand, without the addition of mental challenge, increasing orthostatic loading in our study led to increases in peripheral resistance. This has been previously reported [18]. The increased peripheral resistance contributes to the maintenance of blood pressure during standing (see [14]) which supports cardiovascular regulation leading to the baseline and repeated tolerance times.

\subsection{Delaying the onset of syncope with mental challenge}

The sympathetic nervous system plays an important role in controlling resistance vessels, with an almost maximal activation of control immediately prior to the syncopal attack followed by a complete suppression during and after the syncope.

We hypothesized that the addition of mental challenge may delay the onset of orthostatic syncope. The rationale for this assumption is based on the following observations: A) Sympathetic nervous system activation by mental stress. A beneficial effect of mental challengeinduced increases in sympathetic activity is not only related to change in heart rate or total peripheral resistance: there is also an effect on the venous system. The venous system contains approximately $70 \%$ of total blood volume, and veins are under strong control of the sympathetic nervous system [19]. Being a primary capacitance area of the body, the venous system can exert great changes in venous return and cardiac preload when its capacity is altered. In a previous study, in which we studied the effects of mental challenge on orthostatic responses [4], mental challenge-induced sympathetic activity increased cardiac output, being sustained perhaps by a greater increase in venous tone, increased mean circulatory filling pressure and thus increased venous return (see Guyton's analysis [20]). Our results are supported by observations of Kuipers et al. that mental challenge induces splanchnic vasoconstriction [3]. Therefore, the application of mental challenge shortly before occurrence of presyncopal symptoms and signs should lead to an increase in the orthostatic capacity. B) It is a common observation from clinical practice that engaging persons in mentally challenging activities (conversation) often leads to alleviation of dizziness symptoms, at least temporarily.

\subsection{Timing of mental challenge application}

Hemodynamic responses to stressors are known to reduce over time. Previously, a time dependent decrease in the magnitude of hemodynamic responses to mental [21] and orthostatic challenges [22] has also been reported. How combination of stresses influence cardiovascular variables, however, is poorly understood. We have recently shown that application of mental/orthostatic challenges or combined mental and orthostatic challenges leads to varying cardiovascular responses. We observed that mental stress elicits maximal cardiovascular responses within the first 2-3 min of application [4]. This is important as longer applications result in habituation and adaptation of responses thus minimizing the additive/beneficial effects. Therefore, mental arithmetic was only applied 2-3 min preceding expected presyncopal time.

\subsection{Limitations}

We cannot absolutely rule out the possibility that the increases in orthostatic capacity are due to repetition of the presyncopal runs, especially as literature is divided regarding reproducibility and adaptations to repeated orthostatic stresses. Some investigators reported that subjects adapt to repeated LBNP sessions with increased LBNP tolerance on the third successive exposure [23]. Ector et al. [24], who observed an increase in tolerance times following 1 week of tilt training in patients, also confirmed this. Conversely, applying graded orthostatic stress to the same subjects four times, separated by $72 \mathrm{~h}$ [25] or 2 weeks [10] demonstrated no differences in orthostatic tolerance across trials. Since no objective end point exists for an LBNP tolerance, it is possible that some differences between studies can be attributed to ambiguity associated with LBNP tolerance end point determination [23] or a lay-off effect when tests are more than 1 week apart. For example, aircrews that have not flown at high $G$ for a week develop a reduction in $G$ tolerance on return to flying [26].

We, however, believe that the 2 weeks separation between protocols should suffice to avoid "learning" effects [10]. Furthermore, our experimental protocol with a random order of the last two presyncope sessions (PS + MA versus PS2) was designed to minimize any time-dependent phenomenon. In all five subjects we observed an improvement of the orthostatic tolerance during added mental challenge, regardless of the order of the last two presyncope sessions. Therefore, the observed increases in orthostatic tolerance should be attributable to the effects of mental arithmetic.

\subsection{Clinical and spaceflight applications}

There are several clinical applications of the findings: additional mental loading could be a useful countermeasure to alleviate the orthostatic responses of persons, particularly in those with histories of dizziness on standing up, or to alleviate hypotension that frequently occurs during hemodialysis or on return to earth from the spaceflight environment of microgravity.

\subsection{Future directions}

Further studies are needed to investigate whether the beneficial effects of mental arithmetic on orthostatic syncope extend to larger sample sizes and also to females. In addition, the beneficial effects of mental challenge induced improvements in orthostatic tolerance 
should be studied across different phases of the menstrual cycle. To specifically understand the mechanisms of action of the mental arithmetic induced improvements in orthostatic tolerance, future studies should examine blood flow in the splanchnic region and the brain. Mental arithmetic may influence orthostatic tolerance also by modulation of the vestibular and somatosensory functions in healthy subjects. Therefore, the vestibulo-sympathetic and somatosensory-motor responses should also be analyzed. Modulation of the underlying vagal and sympathetic responses by mental arithmetic in subjects as they reach syncope should be further investigated. Finally, how the mental arithmetic modulates baroreflex should be explored.

\section{Acknowledgments}

We wish to thank the participants for their time and patience. Special thanks go to Dr. Bettina Narrath and Mr. Andreas Jantscher, both of Medical University of Graz, for their excellent assistance.

\section{References}

[1] Morillo CA, Eckberg DL, Ellenbogen KA, Beightol LA, Hoag JB, Tahvanainen KUO, et al. Vagal and sympathetic mechanisms in patients with orthostatic vasovagal syncope. Circulation 1997;96:2509-13.

[2] Carter JR, Kupiers NT, Ray CA. Neurovascular responses to mental stress. J Physiol (London) 2005;564:321-7.

[3] Kuipers NT, Sauder CL, Carter JR, Ray CA. Neurovascular responses to mental stress in the supine and upright postures. J Appl Physiol 2008:104:1129-36.

[4] Lackner HK, Goswami N, Papousek I, Roessler A, Grasser EK, Montani JP, et al. Time course of cardiovascular responses induced by mental and orthostatic challenges. Int J Psychophysiol 2010;75:48-53.

[5] Goswami N, Loeppky JA. Hinghofer-Szalkay H. LBNP: past protocols and technical considerations for experimental design. Aviat Space Environ Med 2008·79:459-71.

[6] Laszlo Z, Rossler A, Hinghofer-Szalkay HG. Cardiovascular and hormonal changes with different angles of head-up tilt in men. Physiol Res 2001;50:71-82.

[7] Grasser EK, Goswami N, Rossler A, Vreckoc K, Hinghofer-Szalkay H. Hemodynamic and neurohormonal responses to extreme orthostatic stress in physically fit young adults. Acta Astronaut 2009;64:688-96.

[8] Gisolf J, Westerhof BE, van Dijk N, Wesseling KH, Wieling W, Karemaker JM. Sublingual nitroglycerin used in routine tilt testing provokes a cardiac outputmediated vasovagal response. JACC 2004;44:588-93.
[9] Stevens PM. Cardiovascular dynamics during orthostasis and influence of intravascular instrumentation. AJCC 1966;17:211-1218.

[10] Goswami N, Lackner HK, Grasser EK, Hinghofer-Szalkay HG. Individual stability of orthostatic tolerance response. Acta Physiol Hung 2009;96:157-66.

[11] Julius S. The blood-pressure seeking properties of the central nervous-system. J Hypertens 1988;6:177-85.

[12] Kamiya K, Iwase S, Michikamia D, Fua Q, Mano T. Muscle sympathetic nerve activity during handgrip and post-handgrip muscle ischemia after exposure to simulated microgravity in humans. Neurosci Lett 2000;280:49-52.

[13] Sweene CA, Bootsma M, Van Bolhuis HH. Different autonomic responses to orthostatic and to mental stress in young normals. Homeostasis 1995;36:287-92.

[14] Goldstein IB, Shapiro D. Cardiovascular responses to mental arithmetic and handgrip during different conditions of postural change. Psychophysiology 1988;25:127-36.

[15] Stephenson RB. Modification of reflex regulation of blood pressure by behavior. Annu Rev Physiol 1984;46:133-42.

[16] Jain D, Shaker SM, Burg M, Wackers FJ, Soufer R, Zaret BL. Effects of mental stress on left ventricular and peripheral vascular performance in patients with coronary artery disease. JACC 1998;31:1314-22.

[17] Halliwill JR, Lawler LA, Eickhoff TJ, Dietz NM, Nauss LA, Joyner MJ. Forearm sympathetic withdrawal and vasodilatation during mental stress in humans. J Physiol 1997;504:211-20.

[18] Grasser E, Goswami N, Hinghofer-Szalkay H. Presyncopal cardiac contractility and autonomic activity in young healthy males. Physiol Res 2009;58:817-26.

[19] Pang CCY. Autonomic control of the venous system in health and disease - effects of drugs. Pharmacol Ther 2001;90:179-230.

[20] Montani JP, Van Vliet BN. Understanding the contribution of Guyton's large circulatory model to long-term control of arterial pressure. Exp Physiol 2009:94:382-8.

[21] Sant'Anna ID, De Sousa EB, De Moraes AV, Loures DL, Mesquita ET, Da Nobrega ACL. Cardiac function during mental stress: cholinergic modulation with pyridostigmine in healthy subjects. Clin Sci 2003;105:161-5.

[22] Sheriff DD, Nadland IH, Toska K. Hemodynamic consequences of rapid changes in posture in humans. J Appl Physiol 2007;103:452-8.

[23] Howden R, Tranfield PA, Lightfoot JT, Brown SJ, Swaine IL. The reproducibility of tol erance to lower-body negative pressure and its quantification. Eur J Appl Physiol $2001 ; 84: 462-8$

[24] Ector H, Reybrouck T, Heidbuchel H, Gewillig M, Van de Werf F. Tilt training: a new treatment for recurrent neurocardiogenic syncope and severe orthostatic intolerance. Pacing Clin Electrophysiol 1998;21:193-6.

[25] Lightfoot JT, Hilton Jr F, Fortney SM. Repeatability and protocol comparability of presyncopal symptom limited lower body negative pressure exposures. Aviat Space Environ Med 1991;62:19-25.

[26] Green NDC. Effects of long-duration acceleration. In: Rainford DJ, DP G, editors. Ernsting's aviation medicine. New York: Edward Arnold; 2006. p. 137-58. 